\title{
HapBead: On-Skin Microfluidic Haptic Interface using Tunable Bead
}

\author{
Teng Han ${ }^{1,2} \quad$ Shubhi Bansal $^{3} \quad$ Xiaochen Shi $^{4} \quad$ Yanjun Chen $^{1} \quad$ Baogang Quan $^{5}$ \\ Feng Tian $^{1,2 *} \quad$ Hongan Wang ${ }^{1,2} \quad$ Sriram Subramanian $^{3}$ \\ ${ }^{1}$ State Key Laboratory of Computer Science and Beijing Key Lab of Human-Computer Interaction, Institute of Software, \\ Chinese Academy of Sciences, Beijing, China \\ ${ }^{2}$ School of Artificial Intelligence, University of Chinese Academy of Sciences, Beijing, China \\ ${ }^{3}$ Department of Informatics, University of Sussex, Brighton, UK \\ ${ }^{4}$ Department of Industrial and Manufacturing Engineering, Penn State University, State College, PA, USA \\ ${ }^{5}$ Laboratory of Microfabrication, Institute of Physics, Chinese Academy of Sciences, Beijing, China \\ \{hanteng, tianfeng, hongan\}@iscas.ac.cn, \{ shubhi.Bansal, Sriram\}@sussex.ac.uk, \\ xus161@psu.edu, cyj123qq@gmail.com,quanbaogang@iphy.ac.cn
}

\begin{abstract}
On-skin haptic interfaces using soft elastomers which are thin and flexible have significantly improved in recent years. Many are focused on vibrotactile feedback that requires complicated parameter tuning. Another approach is based on mechanical forces created via piezoelectric devices and other methods for non-vibratory haptic sensations like stretching, twisting. These are often bulky with electronic components and associated drivers are complicated with limited control of timing and precision. This paper proposes HapBead, a new on-skin haptic interface that is capable of rendering vibration like tactile feedback using microfluidics. HapBead leverages a microfluidic channel to precisely and agilely oscillate a small bead via liquid flow, which then generates various motion patterns in channel that creates highly tunable haptic sensations on skin. We developed a proof-of-concept design to implement thin, flexible and easily affordable HapBead platform, and verified its haptic rendering capabilities via attaching it to users' fingertips. A study was carried out and confirmed that participants could accurately tell six different haptic patterns rendered by HapBead. HapBead enables new wearable display applications with multiple integrated functionalities such as on-skin haptic doodles, mixed reality haptics and visual-haptic displays.
\end{abstract}

\section{Author Keywords}

Haptics, microfluidics, wearable devices, fluid flow

\section{CCS Concepts}

-Human-centered computing $\rightarrow$ Human computer interaction (HCI); Haptic devices;

\footnotetext{
*Corresponding author

Permission to make digital or hard copies of all or part of this work for personal or classroom use is granted without fee provided that copies are not made or distributed for profit or commercial advantage and that copies bear this notice and the full citation on the first page. Copyrights for components of this work owned by others than ACM must be honored. Abstracting with credit is permitted. To copy otherwise, or republish, to post on servers or to redistribute to lists, requires prior specific permission and/or a fee. Request permissions from permissions@acm.org.

CHI'20, April 25-30, 2020, Honolulu, HI, USA

(C) 2020 ACM. ISBN 978-1-4503-6708-0/20/04 . .\$15.00

DOI: https : //doi .org/10.1145/3313831. 3376190
}

\section{INTRODUCTION}

Recent progress in on-skin interfaces tends to be thin and flexible, endowing a range of novel applications such as biointegrated sensing, screening and health monitoring while maintaining a good comfort level [23]. Such interfaces take the benefits of being "soft" instead of "rigid", less obtrusive and not impeding a user's natural dexterity. This is achievable, thanks to the rapid advances in the development of versatile flexible and stretchable electronics, soft materials, microstructural configurations, etc. However, less attention has been paid to the development of on-skin haptic interfaces [44]. This is largely due to the absence of equally reliable, responsive and expressive actuation mechanisms at a small scale and in a flexible formfactor compared with that of rigid mechanisms. Indeed, despite the technical barriers, developing on-skin haptic interfaces is seen to play an important role in the foreseeable age of smart devices [28].

Researchers have been exploring robust haptic rendering methods that can be adapted to the thin and flexible devices. For instance, a common approach is known as electrotactile interface that renders vibration-like sensations via electrostimulation. It is capable of directly activating sensory nerves with electrical current from an electrode on the skin surface [32]. By selecting appropriate voltages and currents it creates desired responses to generate sensations of vibrations, textures and even forces [38]. A notable work is Tacttoo [44], in which the authors recently demonstrated the feasibility of using thin-layered tactors to build ultra-thin tactile interfaces on fingertips. However, the confounding challenge exists on variability of skin impedance across body and between individuals, which requests complicated parameter tuning [51]. Another way is based on mechanical forces created via piezoelectric devices, and other methods such as using shape memory alloy wires $[13,14]$ that have been used to render non-vibratory haptic sensations like squeezing, stretching, dragging, and pulling. These open up a broad range of wearable and epidermal Virtual Reality (VR) interfaces. Though promising, these methods are bulky with components like relays and wires [51], and associated drivers are complicated with limited control of timing and precision. 


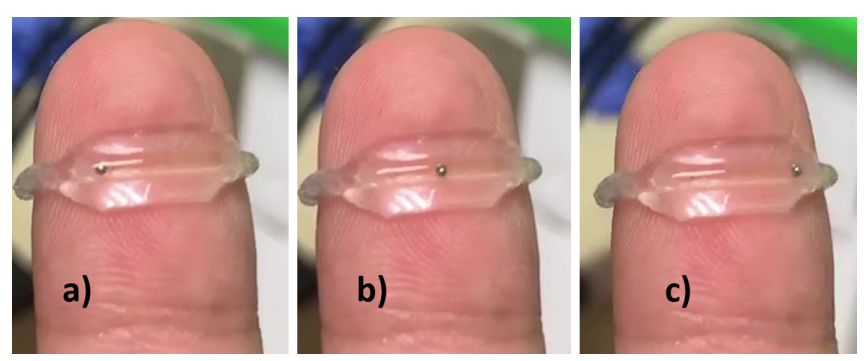

Figure 1. HapBead Interface for three bead positions: at a) left, b) center, and c) right of the channel.

This paper proposes HapBead, a new on-skin haptic interface that is capable of rendering expressive tactile sensations with simpler structure but enhanced tunability. HapBead leverages a microfluidic channel to transport and oscillate a solid bead via liquid flow. By precisely modulating the liquid flow, the bead is oscillated longitudinally at different locations, frequencies and amplitudes. In particular, the liquid flow at micro level $(<1 \mathrm{~mm})$ allows the device to provide precise positioning and targeted control (Figure 1), by allowing a rich set of parameters such as the bead's position, diameter, moving patterns of beads, and bead material for electrical and thermal conductivity etc. The haptic sensation is felt due to both the bead's motion, and localized turbulence caused by its motion in the fluid when the device is softly laminated onto the skin. The tunability of the bead allows the device to produce ondemand and reconfigurable vibration sensations in the channel. Reported literature talks about haptic sensations on full pneumatic [35] or hydraulic circuit [15] via inflation and deflation of the whole fluidic chamber. Here we demonstrated that creating tactile sensations in the flow for different patterns is feasible with a single bead inside the channel. The prototype of HapBead measures i) the flow pressure required to precisely move the bead in a channel, ii) oscillating bead tangentially at different vibration frequencies using inlet and outlet flow pressure, iii) creating bead vibration based fluid pressure for haptic sensation in a micro-channel, and iv) integrating microchannel based on-skin haptic interface on a finger tip.

Microfluidics is the science to move and manipulate fluids and particles at small scales. We used soft materials like SU8 and PDMS, and a standard soft lithography process to fabricate the molds and fluid channel, measured on the millimeter. Due to its soft and flexible nature, the fluid channels of high precision can be easily made thin and attached to a user's body parts, even with large curvature like fingerpad. To work with smaller device size, the flow characteristics like channel dimensions, flow rate and fluid pressure were optimized. This allows the small bead inside the channel to be moved precisely, and with a wide range of manipulating parameters. This paper, for the first time, demonstrates a feasible way to exploit the microfluidic properties for rendering tactile sensations. With a proof-of-concept haptic display that is attached to a user's fingerpad, HapBead enables new applications such as haptic doodles, mixed reality haptics and visual-haptic displays.

We conducted a controlled study to evaluate the prototype and determine whether or not users can perceive and recognize the tactile stimuli of different frequencies and amplitudes pro- duced by the device. Six primary patterns were selected as a part of preliminary evaluation of the current implementation. Results showed that the overall accuracy for recognizing all the patterns was $91.02 \%$, while oscillating the bead fast at the center of the channel, and oscillating the bead slowly and across the channel reached the highest accuracy. The participants found that Hapbead presented overall clear haptic sensations with localized vibrations, and confirmed that bead oscillation with larger amplitudes was more noticeable. The participants also liked the precise haptic rendering capabilities with such a concise formfactor.

This paper made the following contributions: i) the design of HapBead, a novel on-skin haptic interface that exploits motion of a bead in a microfluidic channel; ii) the haptic sensation rendering mechanism via oscillating a physical bead in the micro-channel with control on frequency and amplitude; iii) demonstration of potential applications and unique properties of microfluidic haptic interfaces.

\section{RELATED WORK}

This paper discussed around flexible on-skin haptic interface based on microfluidics, that is inspired by and builds upon prior research in epidermis user interface, wearable haptic display, and microfluidics based flexible sensors and actuators.

\section{Epidermis User Interface}

Skin has been considered as an important next generation interactive surface, that helps in exploring applications beyond the rectangular, planar, and rigid touchscreen technology [16]. Epidermis interfaces bring closer the interactions to the body, weighting future interaction towards being more subtle, intuitive and implicit [8]. Researchers have created novel input and output interface directly on skin to achieve a host of novel applications. For visual display, researchers developed ultrathin flexible LED display that can be attached onto the body, turning the skin into visual displays [50]. Wang et al. [42] demonstrated a fabrication process to create dynamic skin visual appearances by soft fluidic actuation. For interaction, iSkin [43] proposed the idea of adding a very thin sensor overlay on various locations of the body such as the finger, forearm, or ear, turning them into input canvas. Later on, the same team developed a skin overlay with high resolution of touch sensing and supports multi-touch input on the skin [29]. For haptics, Withana et al. [44] worked on adding vibrotactiles to the skin overlay, and Hamdan et al. [14] designed shape memory alloy based skin sticker to produce non-vibrotactile feedback like stretching and pinching. These works define new interface paradigms that could be envisioned with implantable devices.

The development of epidermis interfaces is achievable, thanks to the rapid advances in the development of versatile flexible and stretchable electronics. Soft electronics field has been prevailed for decades with soft, curved, time-dynamic characteristics that enable engineers to measure a broad range of biophysical, biochemical and environmental signals to quantify human performance and supervise overall health status [33]. The soft, bio-integrated sensors embedded in skin-interfaced devices can measure bio-potentials, absolute or relative physical motions and thermal signals associated with activity of 
the heart, peripheral nervous system, brain, skeletal muscles and vascular tree [33]. Biochemical signals such as metabolites (sweat, saliva and tears), electrolytes and miscellaneous biochemical signals are monitored and leveraged to predict human's health condition. Besides, Zhang et al. [52] developed a human motion monitoring in sports using wearable graphene-coated fiber sensors. Krishnan et al. [21] firstly offered wireless, battery-free epidermal electronic device for recording skin thermal properties and hydration state.

HapBead is a novel on-skin haptic interface, relying on an alternative technical approach (i.e., microfluidics) other than the soft electronics.

\section{Wearable Haptic Display}

Wearable haptic devices have been well studied and were showed to be effective in providing localized cutaneous stimuli such as on fingertip [2]. Researchers have been designing and exploring technical solutions to provide cutaneous sensations in multiple dimensions, such as finger worn haptic displays that produce indentation $[9,10,31]$, lateral force [12, 41], and vibration [24]. Soft gloves have been developed using pneumatic actuators and piezosensor sytems to provide tactile feedback $[3,37]$.

In recent years, there has been a growing need to develop wearable haptic devices with compact size, that support, to some extend, real world interactions while worn. AeroFinger [7] was designed with the goal of reducing the physical size of haptic devices, it was a pneumatic device that consisted of four miniature airbags to provide force and tactile sensations to the fingertips. HydroRing [15] was also a fingertip worn device, that took a hydraulic approach and rendered pressure, vibration and temperature sensations. EPFL's SPA-skin [35] used a multilayer quasi 2D manufacturing technique and provided a highly customizable interface with facility of the closed-loop control for multiple vibrotactile signals. Both HydroRing and SPA-skin had similar approach as ours as they are actuated with liquid and air flow, respectively, but HapBead presented a more refined, precise, and localized control provided by an oscillating bead in the channel, not via inflation and deflation on the whole fluidic chamber. Indeed, most of the thin flexible haptic interfaces are developed with vibrotactile feedback $[44,11]$, which is provided generally using piezo-electric and electroactive actuator and sensor systems. Yu et al. [51] presented a wireless, battery-free haptic interface capable of generating spatio-temporally programmable patterns of localized mechanical vibrations. Springlets [14] rendered non-vibro tactile feedback in very thin and flexible formfactor. Hapbead represents an alternative approach for on-skin haptic interface with simpler structure but enhanced tunability.

\section{Microfluidics Based Sensors and Actuators}

The field of microfluidics has grown enormously in past decade, providing integrated platforms with hybrid sensing and actuation mechanisms [5, 17] for numerous applications like fluidic displays [20], opto-fluidic components [36], soft circuit capable of multisignal physiological sensing [47], and lab-on-chip devices [27]. John Rogers' team used soft lithography technique to develop a $900 \mu \mathrm{m}$ thick epidermal microfluidic device for passive sweat capture and analysis for patient populations[54]. Many industrial sectors like Illumina for biological lab-on-chip devices, Fluidwell and liquid-crystal display based firms have launched successful commercial products. However, most of the developed fluid displays are only visual. HapBead is a microfluidic display for haptic feedback. It endows potential applications to not only facilitate impaired and people with disabilities for reading like doodles, but it can also enhance the user experience by coding signals or by changing surface topographies effecting touch gestures associated with the displayed text. The use of a metal bead in the microchannel also allows to develop a closed-loop tactile feedback control by enabling metal-based capacitive and resistive sensing [45, 48].

Researchers have also worked upon microfluidics based tactile sensing [39], however, most of them are based on electrotactile feedback. Lee et al. [40] proposed putting sensing electrodes below the microchannel as the force transducer, where the tactile signal was detected as the impedance change due to the electrodes coverage by the conductive solution. We explore the controlled fluid flow as a mean to actuate beads for haptic sensing. The proposed mechanism does not involve any electronics or complex actuation mechanism debarring the consequences like short-circuit and power consumption. New applications can be integrated with the proposed force mechanism using direct applied pressure, like automated Acupressure therapy by applying strong haptic force.

\section{DESIGN AND IMPLEMENTATION OF HAPBEAD}

We propose HapBead as a new on-skin haptic interface for users to get tactile perception. In this section, we outline important design requirements and implementation of this microfluidics based tactile devices. This helps us to elaborate how the tactile sensation is created. These considerations guided the interfaces presented in the rest of this paper and can also guide future implementations.

\section{Characteristics of Fluid Dynamics in Microchannel}

Different from normal sized tubes, typical microfluidic channel is often of rectangular or circular shape that measures $<1 \mathrm{~mm}$ width [4]. The micro scaled fluid channel allows us to precisely control the inner liquid flow [26]. We leverage this property to develop a haptic rendering mechanism via transporting and oscillating a physical bead in the channel. To design such haptic interface with it and to be capable to apply dynamic control over it, we first have to understand the fluid dynamic properties inside micro channel.

Microfluidic flows in micro-channels are characterized with low Reynold number laminar flows, where the viscous effects are more dominant compared to inertia. Due to this approximation, the complex Navier-Stokes equation is simplified to Hagen Poiseuille flow. The fluidic pressure in the channel is given as - $P=P 1-P 2=R * Q$, where $Q$ is the mean flow-rate, $P=P 1-P 2$ is the pressure drop across the channel and $R$ is the microfluidic resistance [30]. 
The fluid channel with bead consist of three active forces, namely, the hydrostatic pressure, bead drag pressure and the channel-bead wall friction. In the proposed design, controlled flow pressure act as the actuation force. The microfluidic channels were designed with adequate dimensions, to balance the forces for pushing the beads at desired flow rate [49].

Here, the microfluidic resistance changes with the channel dimensions, geometry and the fluid characteristics. For rectangular microfluidic channel, hydraulic resistance is given as [4],

$$
R=\frac{12 \mu L}{w h^{3}\left(1-\frac{h}{w} \frac{192}{\pi^{5}} \sum_{(n=1,3,5)} \frac{1}{n^{5}} \tanh \left(\frac{n \pi w}{2 h}\right)\right)}
$$

where, $\mu$ is the dynamic viscosity, $L$ is the length of microchannel and $d$ is the diameter of micro-channel. We used conventional fluidic models to calculate the channel resistance as $R \approx 10^{8} \mathrm{kgm}^{4} \mathrm{~s}$ approximately. It was used to determine the required flow rate to create a particular pressure drop across the channel. For example, when the applied flow rate was $\approx 10 \mathrm{ml} / \mathrm{s}$, it generated pressure drop of $\Delta P=R * Q \approx 1000 \mathrm{~Pa}$ in the channel, thereby generating $\approx 1 \mathrm{mN}$ force on the bead.

\section{Form Factor}

Smaller bead can be easier to control but may be less effective in generating oscillations. In our implementation, we experimentally determined to use a steel bead with a diameter of $0.9 \mathrm{~mm}$, weighted $2.08 \mathrm{mg}$. This is to make sure haptic sensations can be produced clearly and reliably. The bead of such size also helps maintaining a clear visibility, enabling applications like visual-haptic display that we will demonstrate later. Similar to other on-skin visual and haptic devices $[19,18,25$, 42], the design of HapBead comprises of a two-layer soft plate structure (Figure 2). The bottom layer, measured $0.4 \mathrm{~mm}$ in thickness, embeds the micro channel, whose cross-section is $1 \mathrm{~mm} \times 1 \mathrm{~mm}$. This allows the bead to move freely without arching up the channel wall, while keeping lower gap between the channel and the bead. The top layer is thinner $(0.3 \mathrm{~mm}$ in thickness), bounded to the bottom layer to seal the microchannel. The end-effector of HapBead is about $10 \mathrm{~mm} \times 20 \mathrm{~mm}$ in width and length, and weighted $0.25 \mathrm{~g}$, flexible enough that it can be attached to a user's fingerpad.

The overall thickness of the device is $1.7 \mathrm{~mm}$. This is based on a bottom layer $(0.4 \mathrm{~mm})$ plus a top layer $(0.3 \mathrm{~mm})$ plus a channel $(1 \mathrm{~mm})$. Compared with Sprintlets [14] and Tactoo [44], Hapbead offers expressive sensations with improved precision and control, but it requires a thicker laminated structure, which results in a relatively lower comfort and, to some extend, impedes a user's natural sensations on fingertip (i.e., not feel-through).

\section{Fabrication}

To pattern the channel of the device, we use the collection of techniques referred to as soft lithography [34]. However, due to the design characteristics of the device, there are some changes of fabrication sequence and process. First, The SU-8-Si master was fabricated by photolithography for polydimethylsiloxane (PDMS) micro-channel casting (Figure 3a-e). A 4-inch silicon wafer was coated with SU-8 2150

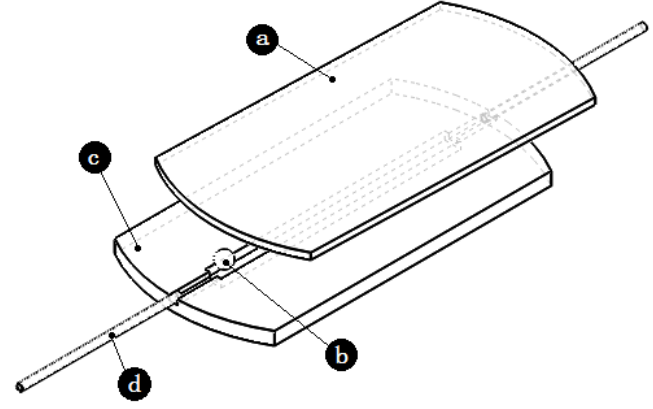

Figure 2. Schematic of device parts: a) PDMS membrane, b)solid bead, c) PDMS substrate, d) liquid flow tube.

photoresist and soft-baked at $65^{\circ} \mathrm{C}$ for 10 minutes and $95^{\circ} \mathrm{C}$ for 2 hours, respectively. The photoresist was in turn patterned using a Karl Suss MA6 Contact Aligner and photomask with a $600 \mathrm{~mJ}$ of UV exposure at $365 \mathrm{~nm}$. A post exposure bake at $65^{\circ} \mathrm{C}$ for 5 minutes and $95^{\circ} \mathrm{C}$ for 30 minutes was applied after exposure. Finally, Patterned wafer was developed in SU-8 developer for 30 minutes.

Second, we used Sylgard PDMS (Dow and Corning's Sylgard 184) with mix ratio of siloxane A cross-linker/curing agent $B$ $=10: 1$ to cast and mould the devices. To ensure thoroughly mixing, the PDMS should be stirred in a flask for at least 5 minutes until it looks milky with tiny air bubbles. The prepared PDMS is then cast into the SU-8-Si master. To make the PDMS clean, transparent and to remove the unwanted air bubbles, the PDMS is degassed in a vacuum desiccator for 10 minutes. After curing in an oven at $85^{\circ} \mathrm{C}$ for 3 hours, PDMS is cured, resulted in a thin layer of PDMS film, and can be peeled away easily from the mold (Figure $3 \mathrm{f}-\mathrm{h}$ ). In so far, the substrate of device is ready and the upper membrane is developed in the same way using a smooth silicon wafer.

As described, we developed a thin PDMS skin with $1.7 \mathrm{~mm}$ thickness $(0.3 \mathrm{~mm}$ thickness of top layer and $1.4 \mathrm{~mm}$ thickness of substrate). Next, the upper layer and the substrate need to be bonded permanently to provide strong barriers against liquid pressure inside (Figure 3i). The PDMS layers were exposed to oxygen plasma $1 \mathrm{~cm}$ on top of surface for 45 seconds for activation. Then the activated PDMS surface is placed on another PDMS layer as soon as possible to form a permanent bond. $55^{\circ} \mathrm{C}$ of post-bake for 4 hours is essential to increase success rate. At last, we cut the device into a flat hexagon and insert one steel bead into the channel(Figure $3 \mathrm{j}$ ). Tygon tubing are adhered to the ports using PDMS and post-bake procedure is carried out to reinforce the joint.

\section{Actuation}

The HapBead prototype consists of a dedicated microfluidic pumping unit (Elveflow ${ }^{1}$, capable to produce $0-2 B A R$ ) and control circuit connected via Tygon tubing to the end-effector attached on a user's fingertip (Figure 4). The pumping unit is programmable and is capable of driving the flow of the liquid precisely. Specifically, The liquid stored in a storage

\footnotetext{
${ }^{1}$ https://www.elveflow.com/
} 


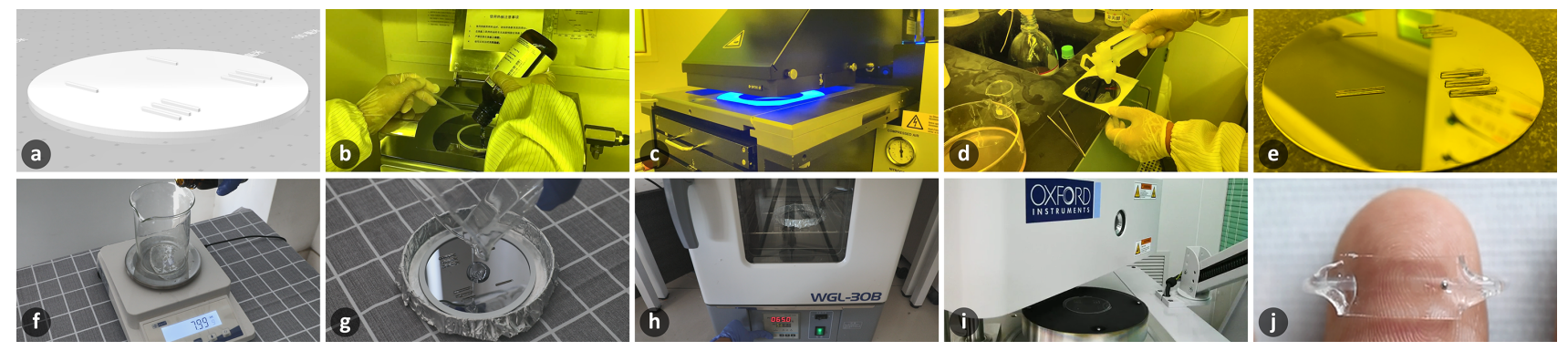

Figure 3. Process of Device fabrication: a) design the mask, b) print mask by spin-coating SU-8 onto the substrate, c) UV exposure and development, d) clean the substrate using deionized water, e) mask forming f) prepare PDMS mix Sylgard 184 at A:B of 10:1, g) pour mixed PDMS into mold, h) degas PDMS in a vacuum desiccator and toast, i) use oxygen plasma etch to activate and bond substrate and membrane permanently, j) cut the bonded device into desired shape, insert steel bead and test the device.
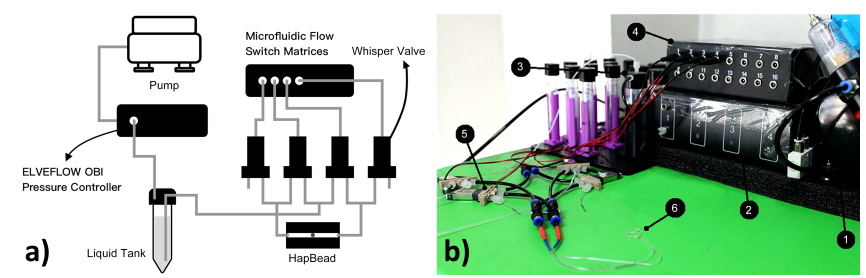

Figure 4. a. Schematic of the control circuit. b. Photo of the control unit: 1) pressure pump, 2) pressure controller, 3)water storage tank, 4) valve controller, 5) three way solenoid valve, 6) HapBead.

tank is pumped to a group of 2 solenoid valves ${ }^{2}$ (DN 1.2 $\mathrm{mm}$, up to $3 \mathrm{bar}$, openning/closing $3 \mathrm{~ms}$ ) connected to the two sides of the end-effector and used to control the inlet of the flow. Another two solenoid valves are used to control the outlet of the flow on both sides. The pressure pump and the solenoid valves are controlled by a Python application with Elveflow Smart Interface SDK ${ }^{3}$, where parameters such as switch of valve, air pressure and frequency change can be controlled precisely. We use water as the liquid medium due to its availability, inert nature and low cost. Experimentally, when the pressure is set at $300-1800 \mathrm{mPa}$ with a $1-200 \mathrm{~Hz}$ frequency, the bead can move and stop instantly. We modulated the pressure and open/close switch through the inlet and outlet, to create tangential oscillations of the bead.

\section{HAPTIC SENSATION AND MOVING PATTERNS OF BEAD}

When the liquid is pushed, the steel bead acts as an obstacle, building up localized pressure inside the channel. The driving pressure is applied at one or another side of the channel for controlling to and fro motion of the bead. The valves control the flow switching in the micro-channel and thus, determines the resulting oscillating frequency of the bead. This oscillating frequency determines the degree of turbulence created in the channel, thereby producing dynamic haptic sensations. We discuss about three main parameters to control the highly tunable haptic feedback, namely, the bead oscillation location, amplitude and frequency. These parameters are manipulated using the driving pressure, the inlet and outlet valves, the channel dimensions and the bead specifications.

\footnotetext{
${ }^{2}$ https://www.burkert.com/en/type/6724

${ }^{3} \mathrm{https}: / /$ www.elveflow.com/microfluidic-flow-control-

products/flow-control-system/elveflow-software/
}

a)

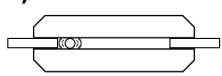

d)

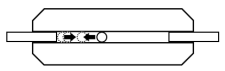

b)

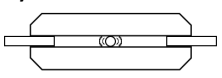

e)

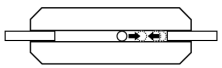

c)

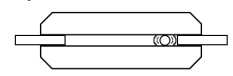

f)

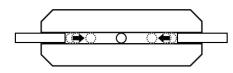

Figure 5. Schematic of different motion patterns of bead for producing different haptic feedback a-c) confined bead oscillation at three positions - left, center and right with very small amplitude; d-e) larger amplitude bead oscillation with the centre of mass before the left and right end of channel. The bead oscillation is confined on one side with the channel walls; f) bead oscillating from left to right over full channel length with center of mass at the center of the channel.

The resulted oscillation of the bead can be measured physically using optical imaging or by involving capacitive sensing for tracking metal bead positon, both of which require sophisticated procedure. Currently, we relied on the microfluidic pumping unit to provide input control of high precision (i.e., pressure, frequency), and captured high quality images for a measurement accuracy of $1 \mathrm{~mm}$ in bead position.

We were able to move the bead in different patterns in the channel by controlling the fluid flow. Here we specified six motion patterns, where we oscillated the bead with different center of mass and motion patterns for generating different haptic feedback (Figure 5). The motion patterns can confine bead oscillation at left, center and right of the channel with tiny amplitude of oscillations, or a larger amplitude bead oscillation can be created with the center of mass between the left/right and center of channel, or bead can be moved from left to right over full channel length with center of mass at the center of the channel. Different patterns have different vibration frequencies: for pattern a-c, the oscillation frequency is around $80 \mathrm{~Hz}$; pattern d-e has $10 \mathrm{~Hz}$; for pattern $\mathrm{f}$ where bead can move the whole channel length, the oscillation is $1 \mathrm{~Hz}$.

\section{USER STUDY: PERCEPTION OF THE HAPTIC PATTERNS}

The six primary patterns were slected as a part of preliminary evaluation of the current implementation. We conducted a user study to determine how well users could differentiate the haptic sensations produced by HapBead after a brief training period. This study serves to validate that the prototype is able 


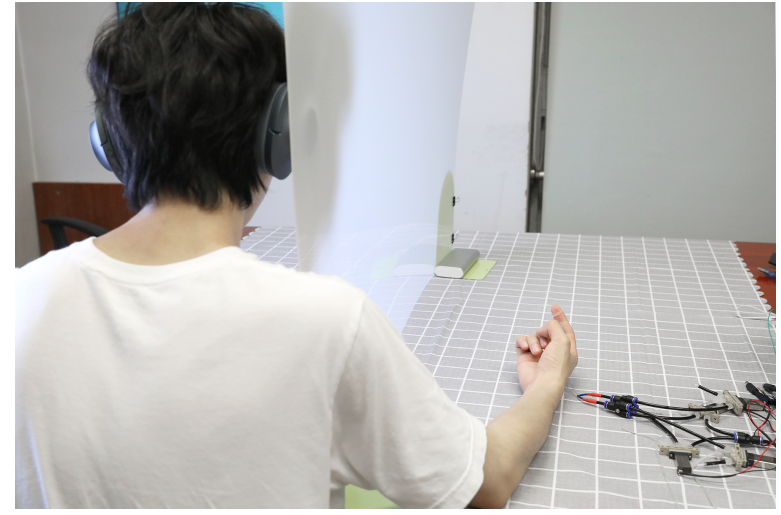

Figure 6. Apparatus for evaluating HapBead. The user's view of the device is occluded, and they wear headphones to mask the noise of the pumps and valves.

to reliably generate haptic sensations and to evaluate users' ability to perceive and recognize the sensations.

\section{Participants}

Twelve (12) participants (5 female, avg. age $=23.9)$ were recruited from a local university for the study. Each participant was rewarded with about \$USD 20 for their participation.

\section{Experimental Apparatus and Procedure}

The participants were first introduced with the purpose of the study and instructed to get familiar with the HapBead prototype. They were seated and rested their hand on a table while the device was lightly stretched over the fingertip of the dominant hand (Figure 6). A laptop computer ran the experimental software, showed feedback to participants, and controlled HapBead.

There were 5 blocks of the test, and in each block, the 6 haptic patterns were repeated 3 times in random order, and the participants were asked which profile they just felt. The participants were asked to take a 1-minute short break after each block. In total, there were 5 blocks $\times 6$ haptic profiles $\times$ 3 repetitions $=90$ trials per participant. The study lasted 40 mins on average.

Once the participants were outfitted with the device, participants began the first block. In each block, they first received 3 minutes training, where they were shown the stimuli in order. Then they were shown the testing set of the stimuli. Each stimuli was played 2 seconds. After each trial, they were asked to verbally report which haptic pattern they just perceived. After the study, the participants were asked to express their subjective opinions about HapBead and the haptic patterns.

\section{Result and Discussion}

The average accuracy was $91.02 \%(\mathrm{SD}=10.2 \%)$ across all the participants and all the haptic patterns. One-way ANOVA yields significant effect between the patterns $\left(F_{5,66}=\right.$ $7.528, p<.01)$. A Tukey post hoc test revealed that the pairs between pattern a and b, a and f, b and c, b and e, c and f, $\mathrm{e}$ and $\mathrm{f}$ were significantly different, while the rest pairs wer not. This can be understood from Figure 7, which shows that pattern $\mathrm{b}$ and $\mathrm{f}$ reached higher accuracy (both $98.89 \%, \mathrm{SD}=$

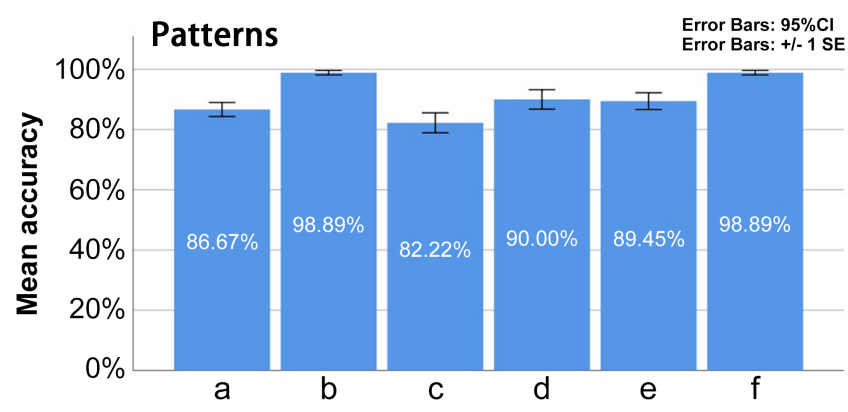

Figure 7. Mean of accuracy by patterns.

\begin{tabular}{|c|c|c|c|c|c|c|}
\hline & $a$ & $b$ & $c$ & $d$ & $e$ & $f$ \\
\hline$a$ & 156 & 0 & 21 & 1 & 2 & 0 \\
\hline$b$ & 2 & 178 & 0 & 0 & 0 & 0 \\
\hline c & 27 & 1 & 149 & 1 & 2 & 0 \\
\hline$d$ & 0 & 0 & 0 & 163 & 16 & 1 \\
\hline e & 2 & 0 & 2 & 9 & 161 & 6 \\
\hline$f$ & 0 & 0 & 0 & 1 & 1 & 178 \\
\hline
\end{tabular}

Figure 8. Confusion matrix of recognition results across the patterns.

$2.6 \%)$ than the others, while pattern a $(86.67 \%, \mathrm{SD}=8.04 \%$ ) and $\mathrm{c}(82.22 \%, \mathrm{SD}=11.49 \%)$ were relatively lower in accuracy. Figure 8 shows a confusion matrix for the patterns we tested. We found that pattern a and c, representing fast bead oscillating frequencies with smaller amplitudes at the left and right side of the channel were more often confused, while the patterns with different bead oscillating frequencies were easy to distinguish. This was probably due to the turbulence caused by the fast but short bead oscillations were weak, thus decreasing the rendered sensations.

The overall performance indicated that the haptic sensations caused by the bead oscillations at various locations in the channel and with different frequencies and amplitudes made clear difference to the participants. Based on the results, we found that with HapBead, the participants were more sensitive to patterns of various vibration frequencies rather than those of various locations. This suggested that designers may consider to leverage a broad range of vibration frequencies to enrich the vocabulary of haptic sensations, while constraining the number of selected locations (e.g., 3). Though the haptic sensations were generally easy to tell, the participants felt a little numb on their fingers after a while. This suggested that designers shall allow the users to not so frequently use the device. We also found that a certain amount of learning helped improve the performance of the participants. HapBead allows designers to create a host of haptic sensations, while how to help users quickly learn the sensations will become challenging. Moreover, we noticed that the device should not be tightly wore by the participants. Otherwise, the external pressure might block the flow. Variability among the haptic perceptions of the participants was obvious. For instance, with pattern d, four participants achieved very high accuracy $(100 \%)$ while two participants only reached $73.33 \%$ 


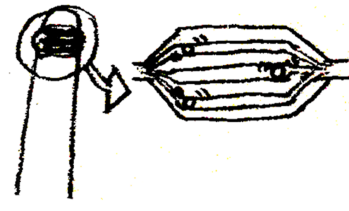

a)

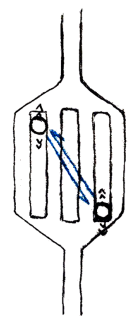

b)

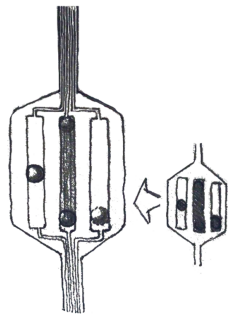

c)
Figure 9. Conceptual scenarios where HapBead is applicable: a)on-skin doodles; b) visuo-haptic display; c) haptic illusion.

and $66.67 \%$, respectively, making the recognition result for the pattern very diverse. According to two participants, they were able to feel the translation of the bead when the the device was rendering the next trial. Though this was not desired, it in fact showed that if improved, the translation motion could be helpful for applications like cutaneous rabbit.

\section{APPLICATIONS}

In this section, we outline three conceptual applications that are feasible for HapBead which emphasize its applicability for novel haptic interfaces.

\section{Application 1: On-Skin Doodles}

The bead flow in straight microfluidic channel produces haptic sensation. The force is controlled by the adhesion-friction forces at bead-channel wall along with the turbulence created by vibrating the bead. We used a single bead microfluidic channel to prove the concept. However, multiple micro-channels can be integrated each with single and multiple bead to display different patterns like Figure 9a. These devices are applicable for developing on-skin doodles and "Dynamic Braille" for visually impaired people.

\section{Application 2: Visuo-Haptic Display}

The bead being silver in colour is readily visible through the transparent microfluidic channel, thereby allowing to integrate the visual feedback along with the haptic sensation. The device can provide both visual and haptic sensation for forming different alphabets and patterns (Figure 9b). Thus, we propose to develop visuo-haptic display using soft on-skin microfluidic channels.

\section{Application 3: Haptic Illusions}

The device can be triggered with different motion patterns at very high speed, to give a sense of apparent motion, in accord to cutaneous rabbit and other haptic illusions. In these illusions, a sequence of taps at two separated skin locations results in the perception that intervening skin regions were also tapped [46]. Hapbead can operate on the same principle and create such haptic illusions (9c).

\section{DISCUSSION AND FUTURE WORK}

We used a bead in a soft microfluidic interface to provide haptic sensation. The use of a bead in microfluidic channel enhances the control of haptic sensation by allowing 1) oscillating the bead in different motion patterns for different vibration frequencies and amplitudes 2) the bead also provide the visual feedback along with the haptic sensation, thus, moving towards mixed reality haptics. The flow in a microfluidic channel was controlled to be a laminar Poiseuille flow. However, by putting a bead in the channel, the turbulence was triggered using the bead oscillations. The bead vibration frequency was controlled to manipulate the amplitude of haptic sensation.

We tried three different fluids, namely, air, oil and water for moving bead into the channel, however, the best control was achieved using water. The air has less viscosity and density than water, thus it applied lesser drag forces to move the bead. Oil also had less density than water, thus it acted more as lubricant with lesser drag force. The different properties of fluids and channel materials like temperature, viscosity, surface tension, composition and conductivity could be explored further for recruiting more useful information.

The channel geometry also plays a significant role while designing HapBead platform. We also tried circular channel geometry for bead motion. However, we observed larger hysteresis/friction loss because of more surface contact area at circular channel-bead wall. While for the rectangular channels, the circular bead had only four point contacts at the horizontal and vertical diameter end points. Thus, rectangular channel showed more efficient bead control with less coagulation and surface friction.

As for the fabrication, we tried but did not choose to 3D print the masks (i.e., using Ultimaker S5) as they cannot provide the same level of qualities, e.g., surface smoothness, high temperature resistance, which are critical for the fabrication of microfluidic channels at that micro scale. In the study, the six primary patterns were selected as a part of preliminary evaluation of the current implementation, but more patterns are possible based on the control of flow rates and channel design. During the study, we also found that the static bead cannot be felt. However, if a user applies some pressure on it (e.g., squeeze with another finger, or on a surface), it can be felt. This provides opportunities for developing static and passive haptic sensations without oscillating the bead, suitable for power efficient scenarios.

In future, the proposed technique could be used to develop "Dynamic Braille" for visually impaired people. The platform could also be modified for portable DIY type fabrication using soft plastic moulds. The automation could be achieved using lab-on-chip fluidic techniques like electrophoresis [22] and electrowetting [1]. Besides, the pumps and associated bulky materials can be miniaturized to wearable fluid platforms by using techniques like self-propelling mechanism [53] or external electric actuation [6]. The device could also be used for point-of-care medical applications like Acupressure therapy without any need of complex electronics. The technique also develops toward enriching user experience by enabling potential future applications like coded-haptic information, 
modulating microfluidic skin for texturing and topographical changes to feel different touch gestures with different texts.

\section{CONCLUSION}

We proposed and successfully demonstrated a microfluidic based on-skin haptic platform, termed here as HapBead.The motion of a solid bead in a thin, flexible and transparent microfluidic circuit, was used to create strong tunable haptic sensation. The pressurized fluid flow generated by tangential oscillation of a bead inserted in channel produced dynamic haptic forces. The tunability of bead and its oscillation amplitude and frequency allowed us to create reconfigurable haptic force. Based on the control of fluid flow, channel and bead dimensions, we developed a on-skin microfluidic haptic platform. HapBead can be applied for on-skin wearable applications like Acupressure therapies, on-skin doodles and displays without any need of complex electronics. Thus, HapBead provides a significant advancement in the field of humancomputer interaction involving microfluidic technology, with a diverge market ranging from on-skin displays to point-of-care health services.

\section{ACKNOWLEDGMENTS}

This project was funded by the National Key R\&D Program of China (2016YFB1001402), the National Natural Science Foundation of China (61972387), the ERC Advanced Grant (787413), as well as the Royal Academy of Engineering (CIET18/19).

\section{REFERENCES}

[1] Shubhi Bansal and Prosenjit Sen. 2016. Mixing enhancement by degenerate modes in electrically actuated sessile droplets. Sensors and Actuators B: Chemical 232 (2016), 318-326.

[2] Carlos Bermejo and Pan Hui. 2017. A survey on haptic technologies for mobile augmented reality. arXiv preprint arXiv: 1709.00698 (2017).

[3] Youngsu Cha, Jeonggyu Seo, Jun-Sik Kim, and Jung-Min Park. 2017. Human-computer interface glove using flexible piezoelectric sensors. Smart Materials and Structures 26, 5 (2017), 057002.

[4] RJ Cornish. 1928. Flow in a pipe of rectangular cross-section. Proceedings of the Royal Society of London. Series A, Containing Papers of a Mathematical and Physical Character 120, 786 (1928), 691-700.

[5] Loïc D'eramo, Benjamin Chollet, Marie Leman, Ekkachai Martwong, Mengxing Li, Hubert Geisler, Jules Dupire, Margaux Kerdraon, Clémence Vergne, Fabrice Monti, and others. 2018. Microfluidic actuators based on temperature-responsive hydrogels. Microsystems \& Nanoengineering 4 (2018), 17069.

[6] Manjeet Dhindsa, Jason Heikenfeld, Seyeoul Kwon, Jungwon Park, Philip D. Rack, and Ian Papautsky. 2010. Virtual electrowetting channels: electronic liquid transport with continuous channel functionality. Lab Chip 10 (2010), 832-836. Issue 7. DOI : http://dx.doi.org/10.1039/B925278A
[7] Yuan-Ling Feng, Charith Lasantha Fernando, Jan Rod, and Kouta Minamizawa. 2017. Submerged Haptics: A 3-DOF Fingertip Haptic Display Using Miniature 3D Printed Airbags. In ACM SIGGRAPH 2017 Emerging Technologies (SIGGRAPH '17). ACM, New York, NY, USA, Article 22, 2 pages. DOI : http://dx.doi.org/10.1145/3084822. 3084835

[8] Jonathan Follett. 2014. Designing for emerging technologies: UX for genomics, robotics, and the internet of things. " O’Reilly Media, Inc.".

[9] Gabriele Frediani, Daniele Mazzei, Danilo Emilio De Rossi, and Federico Carpi. 2014. Wearable wireless tactile display for virtual interactions with soft bodies. Frontiers in bioengineering and biotechnology 2 (2014), 31.

[10] Antonio Frisoli, Massimiliano Solazzi, Fabio Salsedo, and Massimo Bergamasco. 2008. A fingertip haptic display for improving curvature discrimination. Presence: Teleoperators and Virtual Environments 17, 6 (2008), 550-561.

[11] Christian Frisson, Julien Decaudin, Thomas Pietrzak, Alexander Ng, Pauline Poncet, Fabrice Casset, Antoine Latour, and Stephen A. Brewster. 2017. Designing Vibrotactile Widgets with Printed Actuators and Sensors. In Adjunct Publication of the 30th Annual ACM Symposium on User Interface Software and Technology (UIST '17). ACM, New York, NY, USA, 11-13. DOI : http://dx.doi.org/10.1145/3131785.3131800

[12] Brian T Gleeson, Scott K Horschel, and William R Provancher. 2010. Design of a fingertip-mounted tactile display with tangential skin displacement feedback. IEEE Transactions on Haptics 3, 4 (2010), 297-301.

[13] Aakar Gupta, Antony Albert Raj Irudayaraj, and Ravin Balakrishnan. 2017. HapticClench: Investigating Squeeze Sensations Using Memory Alloys. In Proceedings of the 30th Annual ACM Symposium on User Interface Software and Technology (UIST'17). ACM, New York, NY, USA, 109-117. DOI : http://dx.doi.org/10.1145/3126594.3126598

[14] Nur Al-huda Hamdan, Adrian Wagner, Simon Voelker, Jürgen Steimle, and Jan Borchers. 2019. Springlets: Expressive, Flexible and Silent On-Skin Tactile Interfaces. In Proceedings of the 2019 CHI Conference on Human Factors in Computing Systems (CHI '19). ACM, New York, NY, USA, Article 488, 14 pages. DOI : http://dx.doi.org/10.1145/3290605.3300718

[15] Teng Han, Fraser Anderson, Pourang Irani, and Tovi Grossman. 2018. HydroRing: Supporting Mixed Reality Haptics Using Liquid Flow. In Proceedings of the 31st Annual ACM Symposium on User Interface Software and Technology (UIST'18). ACM, New York, NY, USA, 913-925. DOI :

http://dx.doi.org/10.1145/3242587.3242667

[16] Chris Harrison. 2013. The human body as an interactive computing platform. Ph.D. Dissertation. Google. 
[17] Sreenivasa Saravan Kallempudi, Zeynep Altintas, Javed H. Niazi, and Yasar Gurbuz. 2012. A new microfluidics system with a hand-operated, on-chip actuator for immunosensor applications. Sensors and Actuators B: Chemical 163, 1 (2012), 194 - 201. DOI: http://dx.doi.org/https: //doi.org/10.1016/j.snb.2012.01.034

[18] Hsin-Liu (Cindy) Kao, Miren Bamforth, David Kim, and Chris Schmandt. 2018. Skinmorph: Texture-tunable On-skin Interface Through Thin, Programmable Gel. In Proceedings of the 2018 ACM International Symposium on Wearable Computers (ISWC'18). ACM, New York, NY, USA, 196-203. DOI :

http://dx.doi.org/10.1145/3267242. 3267262

[19] Hsin-Liu (Cindy) Kao, Christian Holz, Asta Roseway, Andres Calvo, and Chris Schmandt. 2016. DuoSkin: Rapidly Prototyping On-skin User Interfaces Using Skin-friendly Materials. In Proceedings of the 2016 ACM International Symposium on Wearable Computers (ISWC '16). ACM, New York, NY, USA, 16-23. DOI : http://dx.doi.org/10.1145/2971763.2971777

[20] Kazuhiro Kobayashi and Hiroaki Onoe. 2018. Microfluidic-based flexible reflective multicolor display. Microsystems \& nanoengineering 4, 1 (2018), 17.

[21] Siddharth R Krishnan, Chun-Ju Su, Zhaoqian Xie, Manish Patel, Surabhi R Madhvapathy, Yeshou Xu, Juliet Freudman, Barry Ng, Seung Yun Heo, Heling Wang, and others. 2018. Wireless, Battery-Free Epidermal Electronics for Continuous, Quantitative, Multimodal Thermal Characterization of Skin. Small 14, 47 (2018), 1803192.

[22] Di Li and Xiangchun Xuan. 2018. Electrophoretic slip-tuned particle migration in microchannel viscoelastic fluid flows. Physical Review Fluids 3, 7 (2018), 074202.

[23] Tie Li, Yue Li, and Ting Zhang. 2019. Materials, Structures, and Functions for Flexible and Stretchable Biomimetic Sensors. Accounts of chemical research 52, 2 (2019), 288-296.

[24] Robert W Lindeman, Yasuyuki Yanagida, Haruo Noma, and Kenichi Hosaka. 2006. Wearable vibrotactile systems for virtual contact and information display. Virtual Reality 9, 2-3 (2006), 203-213.

[25] Joanne Lo, Doris Jung Lin Lee, Nathan Wong, David Bui, and Eric Paulos. 2016. Skintillates: Designing and Creating Epidermal Interactions. In Proceedings of the 2016 ACM Conference on Designing Interactive Systems (DIS '16). ACM, New York, NY, USA, 853-864. D0I : http://dx.doi.org/10.1145/2901790.2901885

[26] Nicholas Mavrogiannis, Markela Ibo, Xiaotong Fu, Francesca Crivellari, and Zachary Gagnon. 2016. Microfluidics made easy: A robust low-cost constant pressure flow controller for engineers and cell biologists. Biomicrofluidics 10, 3 (2016), 034107.
[27] Georgia-Paraskevi Nikoleli, Christina G Siontorou, Dimitrios P Nikolelis, Spyridoula Bratakou, Stephanos Karapetis, and Nikolaos Tzamtzis. 2018. Biosensors Based on Microfluidic Devices Lab-on-a-Chip and Microfluidic Technology. In Nanotechnology and Biosensors. Elsevier, 375-394.

[28] Aditya Shekhar Nittala, Klaus Kruttwig, Jaeyeon Lee, Roland Bennewitz, Eduard Arzt, and Jürgen Steimle. 2019. Like A Second Skin: Understanding How Epidermal Devices Affect Human Tactile Perception. In Proceedings of the 2019 CHI Conference on Human Factors in Computing Systems (CHI'19). ACM, New York, NY, USA, Article 380, 16 pages. DOI : http://dx.doi.org/10.1145/3290605.3300610

[29] Aditya Shekhar Nittala, Anusha Withana, Narjes Pourjafarian, and Jürgen Steimle. 2018. Multi-Touch Skin: A Thin and Flexible Multi-Touch Sensor for On-Skin Input. In Proceedings of the 2018 CHI Conference on Human Factors in Computing Systems (CHI '18). ACM, New York, NY, USA, Article 33, 12 pages. DOI : http://dx.doi.org/10.1145/3173574.3173607

[30] Kwang W Oh, Kangsun Lee, Byungwook Ahn, and Edward P Furlani. 2012. Design of pressure-driven microfluidic networks using electric circuit analogy. $L a b$ on a Chip 12, 3 (2012), 515-545.

[31] Claudio Pacchierotti, Stephen Sinclair, Massimiliano Solazzi, Antonio Frisoli, Vincent Hayward, and Domenico Prattichizzo. 2017. Wearable haptic systems for the fingertip and the hand: taxonomy, review, and perspectives. IEEE transactions on haptics 10, 4 (2017), 580-600.

[32] Daniel S Pamungkas and Wahyu Caesarendra. 2018. Overview Electrotactile Feedback for Enhancing Human Computer Interface. In Journal of Physics: Conference Series, Vol. 1007. IOP Publishing, 012001.

[33] Tyler R Ray, Jungil Choi, Amay J Bandodkar, Siddharth Krishnan, Philipp Gutruf, Limei Tian, Roozbeh Ghaffari, and John A Rogers. 2019. Bio-integrated wearable systems: a comprehensive review. Chemical reviews 119 , 8 (2019), 5461-5533.

[34] John A Rogers and Ralph G Nuzzo. 2005. Recent progress in soft lithography. Materials today 8, 2 (2005), 50-56.

[35] Harshal A. Sonar, Aaron P. Gerratt, Stéphanie P. Lacour, and Jamie Paik. 2019. Closed-Loop Haptic Feedback Control Using a Self-Sensing Soft Pneumatic Actuator Skin. Soft Robotics (2019).

[36] Chaolong Song and Say Tan. 2017. A perspective on the rise of optofluidics and the future. Micromachines 8, 5 (2017), 152.

[37] Kahye Song, Sung Hee Kim, Sungho Jin, Sohyun Kim, Sunho Lee, Jun-Sik Kim, Jung-Min Park, and Youngsu Cha. 2019. Pneumatic actuator and flexible piezoelectric sensor for soft virtual reality glove system. Scientific reports 9, 1 (2019), 8988. 
[38] Mayuko Tezuka, Norihide Kitamura, Kohei Tanaka, and Norihisa Miki. 2016. Presentation of various tactile sensations using micro-needle electrotactile display. PloS one 11, 2 (2016), e0148410.

[39] Wei-Yu Tseng, Jeffrey S Fisher, JL Prieto, K Rinaldi, G Alapati, and AP Lee. 2009a. A slow-adapting microfluidic-based tactile sensor. Journal of Micromechanics and Microengineering 19, 8 (2009), 085002 .

[40] W-Y Tseng, J S Fisher, J L Prieto, K Rinaldi, G Alapati, and A P Lee. 2009b. A slow-adapting microfluidic-based tactile sensor. Journal of Micromechanics and Microengineering 19, 8 (jul 2009), 085002. DOI : http://dx.doi .org/10.1088/0960-1317/19/8/085002

[41] Dzmitry Tsetserukou, Shotaro Hosokawa, and Kazuhiko Terashima. 2014. LinkTouch: A wearable haptic device with five-bar linkage mechanism for presentation of two-DOF force feedback at the fingerpad. In 2014 IEEE Haptics Symposium (HAPTICS). IEEE, 307-312.

[42] Yanan Wang, Shijian Luo, Hebo Gong, Fei Xu, Rujia Chen, Shuai Liu, and Preben Hansen. 2018. SKIN+: Fabricating Soft Fluidic User Interfaces for Enhancing On-Skin Experiences and Interactions. In Extended Abstracts of the 2018 CHI Conference on Human Factors in Computing Systems (CHI EA '18). ACM, New York, NY, USA, Article LBW111, 6 pages. DOI : http://dx.doi.org/10.1145/3170427.3188443

[43] Martin Weigel, Tong Lu, Gilles Bailly, Antti Oulasvirta, Carmel Majidi, and Jürgen Steimle. 2015. iSkin: Flexible, Stretchable and Visually Customizable On-Body Touch Sensors for Mobile Computing. In Proceedings of the 33rd Annual ACM Conference on Human Factors in Computing Systems (CHI '15). ACM, New York, NY, USA, 2991-3000. DOI : http://dx.doi.org/10.1145/2702123.2702391

[44] Anusha Withana, Daniel Groeger, and Jürgen Steimle. 2018. Tacttoo: A Thin and Feel-Through Tattoo for On-Skin Tactile Output. In Proceedings of the 31st Annual ACM Symposium on User Interface Software and Technology (UIST'18). ACM, New York, NY, USA, 365-378. DOI :

http://dx.doi.org/10.1145/3242587. 3242645

[45] Ruben D. Ponce Wong, Jonathan D. Posner, and Veronica J. Santos. 2012. Flexible microfluidic normal force sensor skin for tactile feedback. Sensors and Actuators A: Physical 176 (2012), 62-69.
[46] Lei Xiao, Tingwei Zhang, Chen Kun, Jue Zhang, Tian Yue, Fang Fang, and Chen Lihan. 2019. Psychophysics of wearable haptic/tactile perception in a multisensory context. Virtual Reality \& Intelligent Hardware 1, 2 (2019), 185-200.

[47] Sheng Xu, Yihui Zhang, Lin Jia, ..., and John A. Rogers. 2014. Soft Microfluidic Assemblies of Sensors, Circuits, and Radios for the Skin. Science 344, 6179 (2014), $70-74$

https://science.sciencemag.org/content/344/6179/70

[48] Jianzhu Yin, Veronica J. Santos, and Jonathan D. Posner. 2017. Bioinspired flexible microfluidic shear force sensor skin. Sensors and Actuators A: Physical 264 (2017), 289-297.

[49] Ryuji Yokokawa, Yusuke Sakai, Atsuhito Okonogi, Isaku Kanno, and Hidetoshi Kotera. FORCE MEASUREMENT AND MODELING FOR MOTOR PROTEINS BE-TWEEN MICROSPHERE AND MICROFLUIDIC CHANNEL SURFACE. (????).

[50] Tomoyuki Yokota, Peter Zalar, Martin Kaltenbrunner, Hiroaki Jinno, Naoji Matsuhisa, Hiroki Kitanosako, Yutaro Tachibana, Wakako Yukita, Mari Koizumi, and Takao Someya. 2016. Ultraflexible organic photonic skin. Science advances 2, 4 (2016), e1501856.

[51] Xinge Yu, Zhaoqian Xie, Yang Yu, ..., and John A. Rogers. 2019. Skin-integrated wireless haptic interfaces for virtual and augmented reality. Nature 575 (2019), 473-479.

[52] Jinnan Zhang, Yanghua Cao, Min Qiao, Lingmei Ai, Kaize Sun, Qing Mi, Siyao Zang, Yong Zuo, Xueguang Yuan, and Qi Wang. 2018. Human motion monitoring in sports using wearable graphene-coated fiber sensors. Sensors and Actuators A: Physical 274 (2018), 132-140.

[53] Jie Zhang, Rui Guo, and Jing Liu. 2016. Self-propelled liquid metal motors steered by a magnetic or electrical field for drug delivery. J. Mater. Chem. B 4 (2016), 5349-5357. Issue 32. DOI :

http://dx.doi.org/10.1039/C6TB00996D

[54] Yi Zhang, Hexia Guo, Sung Bong Kim, Yixin Wu, Diana Ostojich, Sook Hyeon Park, Xueju Wang, Zhengyan Weng, Rui Li, Amay J Bandodkar, and others. 2019. Passive sweat collection and colorimetric analysis of biomarkers relevant to kidney disorders using a soft microfluidic system. Lab on a Chip 19, 9 (2019), 1545-1555. 